\title{
Successful Treatment of Elderly Diffuse Large B-Cell Lymphoma with Central Nervous System Recurrence by Rituximab, Ranimusutine, Ifosfamide, Procarbazine, Dexamethasone, and Etoposide Therapy
}

\author{
Junya Miyahara', Naoki Takezako ${ }^{1}$, Miyuki Wagatsuma ${ }^{2}$, Kiyoe Midorikawa $^{2}$, Ichiro Fukuda ${ }^{3}$, \\ Satoshi Noto ${ }^{1}$, Ikuo Saito ${ }^{4}$, Kazuaki Yamada ${ }^{2}$, Akiyoshi Miwa $^{5}$, Naohiro Sekiguchi ${ }^{*}$ \\ ${ }^{1}$ Division of Hematology, National Hospital Organization Disaster Medical Center, Tokyo, Japan; ${ }^{2}$ Division of Pathology, National \\ Hospital Organization Disaster Medical Center, Japan; ${ }^{3}$ Division of Radiology, National Hospital Organization Disaster Medical \\ Center, Japan; ${ }^{4}$ Division of Pathology, National Hospital Organization Sagamihara Hospital, Sagamihara, Japan; ${ }^{5}$ Hematology Divi- \\ sion, National Center for Global Health and Medicine, Japan. \\ Email: "nao26@aol.com
}

Received January $11^{\text {th }}, 2013$; revised January $25^{\text {th }}, 2013$; accepted February $4^{\text {th }}, 2013$

\begin{abstract}
The prognosis of CD20-positive (CD20+) diffuse large B-cell lymphoma (DLBCL) with central nervous system (CNS) recurrence is still poor. A standard treatment for CD20+ DLBCL with CNS recurrence in elderly patients has not been established mainly due to adverse effects. We previously reported the efficacy and safety of MIND-E (ranimustine, ifosfamide, procarbazine, dexamethasone, and etoposide) therapy for elderly CD20+ DLBCL patients with CNS recurrence. Here, we report the use of R-MIND-E therapy (rituximab, ranimustine, ifosfamide, procarbazine, dexamethasone and etoposide) in an elderly CD20+ DLBCL patient with CNS recurrence. The patient achieved a complete response according to Revised Response Criteria for Malignant Lymphoma, and treatment-related toxicity was tolerable. R-MIND-E therapy may be a feasible and useful treatment option for elderly CD20+ DLBCL patients with CNS recurrence.
\end{abstract}

Keywords: Diffuse Large B-Cell Lymphoma; Central Nervous System Recurrence; Rituximab; MIND-E

\section{Introduction}

The prognosis of $\mathrm{CD} 20$-positive $(\mathrm{CD} 20+)$ diffuse large B-cell lymphoma (DLBCL) has been improved by combining CHOP therapy (cyclophosphamide, doxorubicin, vincristine, and predonisone) and rituximab, a chimeric monoclonal antibody against the CD20 B-cell antigen [1]. However, the prognosis of CD20+ DLBCL with central nervous system (CNS) recurrence is still poor [2]. Whole brain irradiation or high dose chemotherapy including methotrexate (MTX) and cytarabine is useful for younger patients; however, these therapies are not appropriate for elderly CD20+ DLBCL patients with CNS recurrence because of treatment-related toxicities including leukoencephalopathy. We previously reported the efficacy and safety of MIND-E therapy (ranimustine (MCNU), ifosfamide (IFO), procarbazine (PCZ), dexamethasone (DEX), and etoposide (ETP)) (Table 1(a)). On the other hand, we did not use rituximab in the study [3]. Recently, regard-

"Corresponding author. ing primary CNS lymphoma (PCNSL), intravenous rituximab in addition to combined chemotherapy has been accepted as the standard treatment strategy [4-7]. Furthermore, CNS recurrence of systemic DLBCL is usually premonitory symptom of systemic recurrence, therefore, administration of rituximab in the salvage therapy may be considered as prevention for systemic reoccurrence. Here, we report a case of an elderly CD20+ DLBCL patient with CNS recurrence treated with R-MIND-E therapy.

\section{Case Report}

A 69-year-old male was admitted to our hospital in March 2010 because of right hemilateral sensory disturbance, short-term memory disturbance, alexia, and agraphia. He had been diagnosed with $\mathrm{CD} 20+$ primary testicular DLBCL in 2005, and underwent orchidectomy, 6 cycles of CHOP therapy, irradiation, and intrathecal injection for CNS prophylaxis. He achieved a complete response (CR). T2 weighted magnetic resonance imaging 
Table 1. (a) Regimen of MIND-E therapy; (b) Regimen of R-MIND-E therapy.

(a)

\begin{tabular}{|c|c|c|c|c|c|c|}
\hline & Dose & Day 1 & Day 2 & Day 3 & Day 4 & Day 5 \\
\hline Ranimustine & $50 \mathrm{mg} / \mathrm{m}^{2} \mathrm{div}$ & o & & & & \\
\hline Ifosfamide & $500 \mathrm{mg} / \mathrm{m}^{2} \mathrm{div}$ & $\circ$ & $\circ$ & $\circ$ & ० & ० \\
\hline Procarbazine & $80 \mathrm{mg} / \mathrm{m}^{2}$ po & ○ & ० & ○ & ० & $\circ$ \\
\hline Etoposide & $100 \mathrm{mg} / \mathrm{m}^{2} \mathrm{div}$ & ० & $\circ$ & $\circ$ & $\circ$ & ० \\
\hline Dexamethasone & $40 \mathrm{mg} /$ body div & $\circ$ & ० & ० & ० & ○ \\
\hline
\end{tabular}

(b)

\begin{tabular}{|c|c|c|c|c|c|c|c|}
\hline & Dose & Day 1 & Day 2 & Day 3 & Day 4 & Day 5 & Day 6 or 7 \\
\hline Ranimustine & $50 \mathrm{mg} / \mathrm{m}^{2} \mathrm{div}$ & ○ & & & & & \\
\hline Ifosfamide & $500 \mathrm{mg} / \mathrm{m}^{2} \mathrm{div}$ & ० & ० & ० & ○ & ○ & \\
\hline Procarbazine & $100 \mathrm{mg} / \mathrm{m}^{2}$ po & $\circ$ & $\circ$ & ० & ○ & $\circ$ & \\
\hline Etoposide & $80 \mathrm{mg} / \mathrm{m}^{2} \mathrm{div}$ & $\circ$ & ○ & ० & ○ & $\circ$ & \\
\hline Dexamethasone & $40 \mathrm{mg} /$ body div & $\circ$ & ० & $\circ$ & $\circ$ & $\circ$ & \\
\hline Rituximab & $375 \mathrm{mg} / \mathrm{m}^{2} \mathrm{div}$ & & & & & & ○ \\
\hline
\end{tabular}

po: per os; div: drip infusion of vein.

(MRI) revealed a high-intensity mass in the left temporal lobe to parietal lobe (Figure 1(a)). Laboratory results were as follows: WBC $5900 / \mu \mathrm{L}, \mathrm{Hb} 12.8 \mathrm{mg} / \mathrm{dL}$, and LDH $209 \mathrm{IU} / \mathrm{L}$. The total cell count in the cerebrospinal fluid (CSF) was $63 / 3 \mu \mathrm{L}$. May-Giemsa staining of the CSF smear showed the diffuse infiltration of atypical large lymphocytes (Figure 2). Therefore, he was diagnosed with CNS recurrence of DLBCL.

He was evaluated to be a poor candidate for whole brain irradiation and high-dose chemotherapy because of his age and poor performance status. Therefore, he was treated with MIND-E therapy. After 4 cycles of MIND-E therapy, MRI (Figure 1(b)) and [18F]-fluorodeoxyglucose positron emission tomography (PET) scans showed no evidence of a tumor, and all detectable clinical evidence of disease and disease-related symptoms disappeared; thus, he achieved CR according to Revised Response Criteria for Malignant Lymphoma [8]. Six months later, MRI revealed second CNS recurrence (Figure 1(c)). At that time, he was treated with 3 cycles of RMIND-E therapy (Table 1(b)). One cycle of R-MIND-E therapy consisted of the intravenous administration of 50 $\mathrm{mg} / \mathrm{m}^{2} \mathrm{MCNU}$ on day 1 , intravenous administration of $500 \mathrm{mg} / \mathrm{m}^{2}$ IFO on days $1-5$, oral administration of 100 $\mathrm{mg} / \mathrm{m}^{2} \mathrm{PCZ}$ on days $1-5$, intravenous administration of $80 \mathrm{mg} / \mathrm{m}^{2}$ ETP on days $1-5$, intravenous administration of $40 \mathrm{mg} /$ body DEX on days 1 - 5, and intravenous administration of $375 \mathrm{mg} / \mathrm{m}^{2}$ rituximab on day 6 or 7 . After 3 cycles of R-MIND-E therapy, no evidence of the tumor was found including MRI (Figure 1(d)) and PET scans, therefore, he achieved a 3rd CR according to Revised

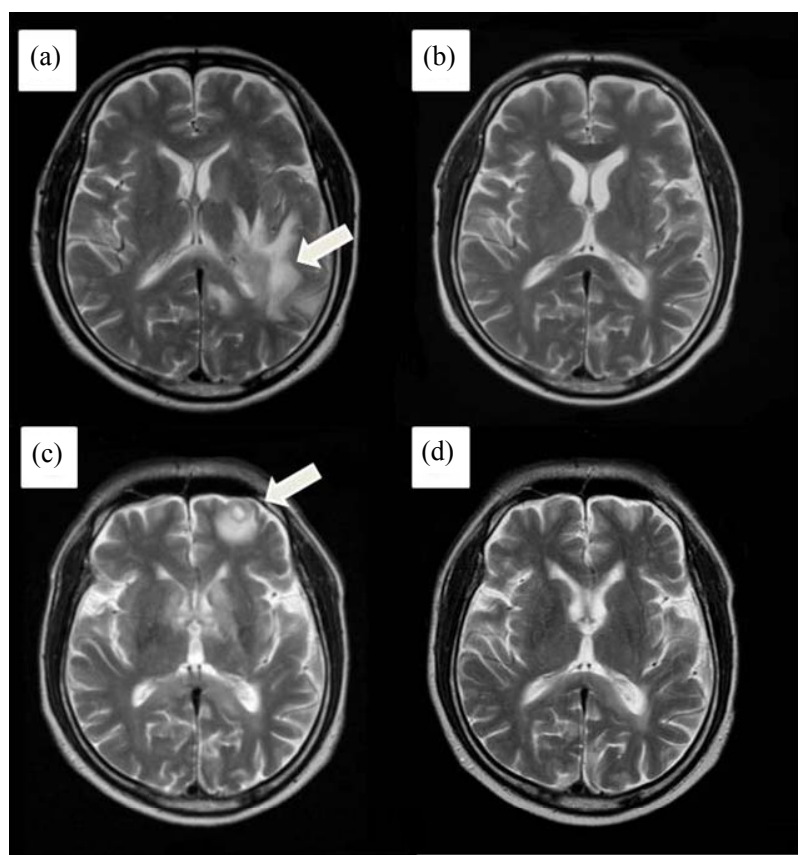

Figure 1. (a) High-intensity mass in the left temporal lobe to parietal lobe on T2 weighted MRI (Arrow); (b) T2 weighted MRI after four cycles of MIND-E therapy; (c) Second CNS recurrence in the left frontal lobe on T2 weighted MRI (Arrow); (d) T2 weighted MRI after three cycles of $\mathrm{R}$ MIND-E therapy.

Response Criteria for Malignant Lymphoma [8]. Grade 4 neutropenia, Grade 3 thrombocytopenia, and Grade 3 infection were observed according to the Common Terminology Criteria for Adverse Events version 4.0. CR has 


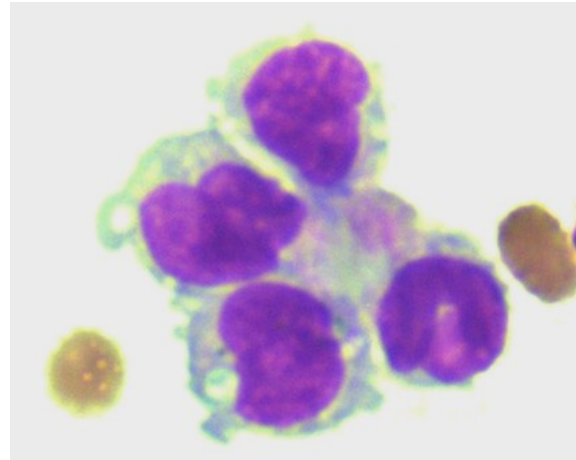

Figure 2. Invasion of atypical large lymphocytes in the cerebrospinal fluid (original magnification $\times \mathbf{1 0 0 0}$ ). The size of atypical lymphocytes was double to triple as big as erythrocytes.

been maintained for 4 months.

\section{Discussion}

The prognosis of systemic DLBCL has improved with the use of rituximab [1]. However, CNS recurrence occurs in $1.1 \%$ to $10.4 \%$ of patients, and the prognosis remains poor [2]. Whether the addition of rituximab decreases the risk of CNS recurrence for systemic DLBCL has not yet been proven [9-12]. The concentration of rituximab in the CSF is low even after its intravenous infusion. Some reports have suggested that a high-dose intravenous rituximab infusion or intrathecal rituximab infusion may be an effective treatment option [13], although the clinical safety of such treatments has not been yet been confirmed. In order to prevent CNS recurrence of DLBCL, intrathecal chemotherapy such as MTX and cytarabine is currently performed in many institutions, although its efficacy has been controversial [2,14-18].

Kim et al. described the treatment of 73 DLBCL patients with secondary CNS involvement in 11 institutions in Korea [14]. They reported that high-dose MTX or localized CNS-directed therapy such as whole brain irradiation was favorable for DLBCL patients with isolated secondary CNS involvement. However, they also reported that the prognosis of DLBCL patients with secondary CNS involvement was still poor, systemic recurrence occurred, and no effective treatment had been established yet. Furthermore, elderly patients who received whole brain irradiation had an increased risk of treatment-related neurotoxicity $[19,20]$, and high-dose chemotherapy was also not feasible for these patients due to a poor performance status or disturbance of consciousness. Therefore, no effective treatment for elderly DLBCL patients with CNS recurrence had been established.

We previously reported on the use of MIND-E therapy in 8 elderly DLBCL patients with CNS recurrence. The 5 drugs used in MIND-E therapy consist of drugs that cross the blood-brain barrier or blood-retina barrier to some extent. Of the 8 patients, three achieved CR, two patients achieved a partial response, and no treatment-related mortality (TRM) was observed [3]. On the other hand, this regimen did not include rituximab; therefore, in the present case, we administered rituximab in addition to MIND-E therapy. The patient achieved a third CR after R-MIND-E therapy, and treatment-related toxicities were tolerable. Although reports concerning the treatment and response of elderly CD20+ DLBCL patients with CNS recurrence remain limited, we successfully achieved CR using R-MIND-E therapy without whole brain irradiation or high-dose chemotherapy.

To the best of our knowledge, regarding the treatment of primary CNS lymphoma, addition of rituximab for primary CNS lymphoma may be considered as standard therapy. Bimbaum et al. recently reported the benefits of rituximab in addition to MTX and IFO over MTX and IFO in 36 cases of primary CNS lymphoma in a retrospective analysis [6]. In prospective study, some investigators described promising response rate and survival in primary CNS lymphoma by multi-agent chemotherapy including rituximab as induction therapy $[4,5,7]$. Therefore, intravenous administration of rituximab for CNS recurrence in systemic DLBCL may be considered as a treatment option.

In conclusion, R-MIND-E therapy may be an effective and safe treatment option for elderly CD20+ DLBCL patients with CNS recurrence. However, a prospective study should be carried out to confirm the efficacy and safety of R-MIND-E therapy for elderly CD20+ DLBCL patients with CNS recurrence.

\section{REFERENCES}

[1] B. Coiffier, E. Lepage, J. Briere, et al., "CHOP Chemotherapy Plus Rituximab Compared with CHOP Alone in Elderly Patients with Diffuse Large B Cell Lymphoma," New England Journal of Medicine, Vol. 346, No. 4, 2002, pp. 235-242. doi:10.1056/NEJMoa011795

[2] W. M. Tai, J. Chung, P. L. Tang, et al., "Central Nervous System (CNS) Relapse in Diffuse Large B Cell Lymphoma (DLBCL): Pre- and Post-Rituximab," Annals of Hematology, Vol. 90, No. 7, 2011, pp. 809-818. doi:10.1007/s00277-010-1150-7

[3] A. Miwa, N. Sekiguchi, A. Tanimura, et al., "Ranimustine, Ifosfamide, Procarbazine, Dexamethasone, and Etoposide Therapy for Central Nervous System Recurrence of Diffuse Large B-Cell Lymphoma in Patients with Poor Performance Status: A Pilot Study," Leukemia and Lymphoma, Vol. 52, No. 10, 2011, pp. 1898-1903. doi:10.3109/10428194.2011.588759

[4] G. D. Shah, J. Yahalom, D. D. Correa, et al., "Combined Immunochemotherapy with Reduced Whole-Brain Radiotherapy for Newly Diagnosed Primary CNS Lymphoma," 
Journal of Clinical Oncology, Vol. 25, No. 30, 2007, pp. 4730-4735. doi:10.1200/JCO.2007.12.5062

[5] R. C. Curry, D. Correa, J. J. Raizer, et al., "Consolidation Reduced Dose Whole Brain Radiotherapy (rdWBRT) Following Methotrexate, Rituximab, Procarbazine, Vincristine, Cytarabine (R-MPV-A) for Newly Diagnosed Primary CNS Lymphoma (PCNSL): Final Results and Long-Term Outcome," Journal of Clinical Oncology, Vol. 30, No. 15, 2012, p. 2006.

[6] T. Bimbaum, E. A. Stadler, L. V. Baumgarten and A. Straube, "Rituximab Significantly Improves Complete Response Rate in Patients with Primary CNS Lymphoma," Journal of Neurooncology, Vol. 109, No. 2, 2012, pp. 285-291. doi:10.1007/s11060-012-0891-7

[7] M. J. Wieduwilt, F. Valles, S. Issa, et al., "Immunochemotherapy with Intensive Consolidation for Primary CNS Lymphoma: A Pilot Study and Prognostic Assessment by Diffusion-Weighted MRI," Clincal Cancer Research, Vol. 18, No. 4, 2012, pp. 1146-1155. doi:10.1158/1078-0432.CCR-11-0625

[8] B. D. Cheson, B. Pfistner, M. E. Juweid, et al., "Revised Response Criteria for Malignant Lymphoma," Journal of Clinical Oncology, Vol. 25, No. 5, 2007, pp. 579-586. doi:10.1200/JCO.2006.09.2403

[9] D. Villa, J. M. Connors, T. N. Shenkier, et al., "Incidence and Risk Factors for Central Nervous System Relapse in Patients with Diffuse Large B-Cell Lymphoma: The Impact of the Addition of Rituximab to CHOP Chemotherapy," Annals of Oncology, Vol. 21, No. 5, 2010, pp. 1046-1052. doi:10.1093/annonc/mdp432

[10] P. Feugier, J. M. Virion, H. Tilly, et al., "Incidence and Risk Factors for Central Nervous System Occurrence in Elderly Patients with Diffuse Large-B-Cell Lymphoma: Influence of Rituximab," Annals of Oncology, Vol. 15, No. 1, 2004, pp. 129-133. doi:10.1093/annonc/mdh013

[11] Y. Shimizu, K. Notohara and Y. Ueda, "Diffuse Large B-Cell Lymphoma with Central Nervous System Relapse: Prognosis and Risk Factors According to Retrospective Analysis from a Single-Center Experience," International Journal of Hematology, Vol. 89, No. 5, 2009, pp. 577583. doi:10.1007/s12185-009-0289-2

[12] R. Kridel and P. Dietrich, "Prevention of CNS Relapse in Diff Use Large B-Cell Lymphoma," The Lancet Oncol- ogy, Vol. 12, No. 13, 2011, pp. 1258-1266. doi:10.1016/S1470-2045(11)70140-1

[13] A. Harjunpaa, T. Wiklund, J. Collan, et al., "Complement Activation in Circulation and Central Nervous System after Rituximab (Anti-CD20) Treatment of B-Cell Lymphoma," Leukemia and Lymphoma, Vol. 42, No. 4, 2001, pp. 731-738. doi:10.3109/10428190109099335

[14] S. J. Kim, S. Y. Oh, J. S. Kim, et al., "Secondary Central Nervous System (CNS) Involvement in Patients with Diffuse Large B-Cell Lymphoma: A Therapeutic Dilemma," Annals of Hematology, Vol. 90, No. 5, 2011, pp. 539-546. doi:10.1007/s00277-010-1104-0

[15] A. Kumar, A Vanderplas, A. S. LaCasce, et al., "Lack of Benefit of Central Nervous System Prophylaxis for Diffuse Large B-Cell Lymphoma in the Rituximab Era," Cancer, Vol. 118, No. 11, 2012, pp. 2944-2951. doi: $10.1002 /$ cncr. 26588

[16] N. Schmitz, S. Zeynalova, M. Loeffler, et al., "Response: Intrathecal Methotrexate and Central Nervous System Events," Blood, Vol. 114, No. 9, 2009, pp. 1999-2000. doi:10.1182/blood-2009-06-226076

[17] J. S. Abramson, M. Hellmann, J. A. Barnes, et al., "Intravenous Methotrexate as Central Nervous System (CNS) Prophylaxis Is Associated with a Low Risk of CNS Recurrence in High-Risk Patients with Diffuse Large B-Cell Lymphoma," Cancer, Vol. 116, No. 18, 2010, pp. 42834290. doi:10.1002/cncr.25278

[18] Q. A. Hill and R. G. Owen, "CNS Prophylaxis in Lymphoma: Who to Target and What Therapy to Use," Blood Reviews, Vol. 20, No. 6, 2006, pp. 319-332. doi:10.1016/j.blre.2006.02.001

[19] N. D. Doolittle, L. E. Abrey, T. N. Shenkier, et al., "Brain Parenchyma Involvement as Isolated Central Nervous system Relapse of Systemic Non-Hodgkin Lymphoma an International Primary CNS Lymphoma Collaborative Group Report," Blood, Vol. 111, No. 3, 2008, pp. 10851093. doi:10.1182/blood-2007-07-101402

[20] A. M. P. Omuro, L. M. DeAngelis, Yahalom J., et al., "Chemoradiotherapy for Primary CNS Lymphoma an Intent-to-Treat Analysis with Complete Follow-Up," Neurology, Vol. 64, No. 1, 2005, pp. 69-74. doi:10.1212/01.WNL.0000148641.98241.5E 